\title{
Intramedullary Spinal Cord Metastases: Visibility on PET and Correlation with MRI Features
}

\author{
P.M. Mostardi, F.E. Diehn, J.B. Rykken, L.J. Eckel, K.M. Schwartz, T.J. Kaufmann, C.P. Wood, J.T. Wald, and C.H. Hunt
}

\begin{abstract}
BACKGROUND AND PURPOSE: Studies systematically evaluating the detection of intramedullary spinal cord metastasis with PET are lacking. Our purpose was to evaluate the visibility of intramedullary spinal cord metastasis on PET in a single institutional series and to correlate PET and MR imaging features.
\end{abstract}

MATERIALS AND METHODS: Patients were included if pretreatment MR imaging identifying an intramedullary spinal cord metastasis and an $\left[{ }^{18} \mathrm{~F}\right]$ FDG-PET examination near the time of MR imaging were available. PET examinations were retrospectively reviewed, with reviewers blinded and then unblinded to the PET report and MR imaging findings. PET intramedullary spinal cord metastasis features were compared with and correlated with previously analyzed MR imaging lesion characteristics. Original clinical PET reports were reviewed.

RESULTS: The final study sample was 10 PET examinations in 10 patients with 13 intramedullary spinal cord metastases. In 7 (70\%) patients, retrospective blinded review demonstrated convincing evidence of $10(77 \%)$ intramedullary spinal cord metastases. Three MR imaging features correlated with intramedullary spinal cord metastases being visible on PET compared with those nonvisible, respectively: larger lesion enhancement size: mean size: $32.1 \mathrm{~mm}$ versus $6.0 \mathrm{~mm}(P=.038)$; larger longitudinal extent of T2 signal abnormality: mean 5.6 versus 1.0 segments $(P=.0081)$; and larger ratio of extent of T2 signal abnormality to contrast enhancement: 3.8 versus $1.0(P=.0069)$. Intramedullary spinal cord metastasis was confidently reported clinically in 2 (20\%) patients, accounting for 5 (38\%) intramedullary spinal cord metastases.

CONCLUSIONS: Most intramedullary spinal cord metastases can be detected on PET when performed near the time of pretreatment MR imaging. However, intramedullary spinal cord metastases may not be clinically reported on PET. Larger lesions with more edema are more likely to be visible. The spinal cord should be specifically and carefully assessed on PET for evidence of intramedullary spinal cord metastases to provide timely diagnosis.

ABBREVIATIONS: ISCM = intramedullary spinal cord metastasis; $\left[{ }^{18} \mathrm{~F}\right]=$ fluorine $18 ;$ SUVmax = maximum standardized uptake value

ntramedullary spinal cord metastases (ISCMs) are rare and devastating manifestations of metastatic neoplasm. They are present at autopsy in $0.9-2.1 \%$ of patients with cancer $^{1,2}$ and comprise $1-4 \%$ of spinal metastases. ${ }^{1-3}$ Early detection is important because ISCMs indicate a poor prognosis, and treatment may slow neurologic deterioration. ${ }^{4}$ However, ISCMs can be asymptomatic, especially at early stages, and are historically difficult to

Received March 8, 2013; accepted after revision April 13.

From the Mayo Medical School (P.M.M.), and Division of Neuroradiology (F.E.D., L.J.E., K.M.S., T.J.K., C.P.W., J.T.W., C.H.H.), Department of Radiology, Mayo Clinic, Rochester, Minnesota; and Division of Neuroradiology (J.B.R.), Department of Radiology, University of Minnesota, Minneapolis, Minnesota.

Paper previously presented at: Annual Symposium of the American Society of Spine Radiology, February 2013; Scottsdale, Arizona.

Please address correspondence to Felix E. Diehn, MD, 200 1st St SW, Rochester, MN 55905; e-mail: Diehn.felix@mayo.edu

Indicates article with supplemental on-line tables.

http://dx.doi.org/10.3174/ajnr.A3618 detect with imaging. ${ }^{3,5} \mathrm{MR}$ imaging is the cornerstone of spinal cord imaging, ${ }^{6}$ but this technique is generally not indicated for screening of asymptomatic patients.

$\left[{ }^{18} \mathrm{~F}\right]$ FDG-PET is the primary imaging technique used for whole-body screening for metastases and therefore is a technique that ideally locates most sites of metastases, including intraspinal. However, there are only scattered case reports of visualization of ISCMs on PET. ${ }^{7-12}$ No large series systematically evaluating the detection of ISCMs with PET have been reported. The purpose of the current study was to retrospectively evaluate the visibility of ISCMs on PET in a single institutional series of patients with ISCMs, and to correlate PET and MR imaging features.

\section{MATERIALS AND METHODS}

Institutional review board approval with waived consent was obtained for this Health Insurance Portability and Accountability Act-compliant retrospective research study. 


\section{Subjects}

A search of the radiologic and clinical databases at our institution from 1999-2011 had previously yielded a group of 45 patients with 64 ISCMs, in whom pretreatment MR imaging had identified the ISCM. ${ }^{13}$ For inclusion in the current study, this group was refined to those patients in whom a PET scan (either PET/CT or PET only) was also available for electronic review. It was assumed that the number of potential cases of ISCM seen on PET at our institution but not imaged with MR imaging (and thus not included in our 45-patient cohort) would be negligible or nonexistent, given that patients would be expected to undergo spinal MR imaging if an ISCM was first suggested at PET. When multiple PET examinations were available for a patient, only the examination closest to the MR imaging was included in the study. PET examinations performed more than 60 days before or more than 14 days after the pretreatment diagnostic MR imaging were excluded. The purpose of these criteria was to exclude PET examinations that were estimated to have a high likelihood of predating the development of an ISCM (those performed more than 60 days before MR imaging) and to exclude PET examinations in which the PET appearance probably would have been affected by the preceding treatment of an ISCM (those performed more than 14 days after MR imaging).

\section{PET Acquisition}

Although there was some variability in PET scanner technology, given the more than 10-year time period over which the imaging was performed, our standard $\left[{ }^{18} \mathrm{~F}\right]$ FDG-PET protocol was followed for patient preparation and imaging. Patient preparation included 4 hours of fasting, such that the finger-stick blood glucose level was in the desired range, between $70-180 \mathrm{mg} / \mathrm{dL}$, before injection of the $\left[{ }^{18} \mathrm{~F}\right]$ FDG radiotracer. The injected activity: was $15 \mathrm{mCi}$ of $\left[{ }^{18} \mathrm{~F}\right]$ FDG $( \pm 10 \%)$, with an uptake time of $60-70$ minutes.

All PET images were obtained on General Electric scanners (GE Healthcare, Milwaukee, Wisconsin). Because of the evolving nature of our clinical practice over the course of the study, the scanner types changed with emerging technology. Only 1 of the patients in this study was imaged on a PET only scanner (Advance NX series). The other 9 patients were all imaged on PET/CT scanners: 3 on Discovery 690 series, 1 on Discovery ST series, 2 on Discovery RX series, and 3 on Discovery DLS series.

PET/CT acquisition parameters were helical scan, 0.5 seconds rotation time; pitch, 0.984; table speed, $39.37 \mathrm{~mm} /$ rotation; section thickness, $3.75 \mathrm{~mm}$; $\mathrm{kVp}$ 120. A 3D PET acquisition toward the feet was used with bed position of 3 minutes.

\section{Image Review}

Two radiologists (F.E.D., neuroradiology faculty member with an American Board of Radiology certification and a Certificate of Added Qualification in neuroradiology; C.H.H., neuroradiology and nuclear medicine faculty member with an American Board of Radiology certification, Certificate of Added Qualification-eligible for neuroradiology and nuclear medicine) and a 4th-year medical student (P.M.M.) retrospectively reviewed the PET examinations for evidence of ISCMs in multiplanar fashion on an electronic workstation in consensus fashion. The reviewers were first blinded and, after complete initial review of the PET examinations, subsequently unblinded to the clinical PET report and all MR imaging findings. When concurrent CT was available, images were reviewed both without and with the CT data. Multiple characteristics were analyzed on PET for ISCMs that were identified: lesion location (spinal levels), maximal superior-inferior extent (number of vertebral body segments), morphology of FDG uptake (fusiform versus round), and maximum standardized uptake value (SUVmax) of the ISCM. SUVmax of the mediastinal blood pool was measured on all PET examinations. The SUVmax of both the ISCMs and of the mediastinal blood pool was determined by placing a circular region of interest over the area that included the subjective maximum uptake. Several MR imaging lesion characteristics previously analyzed by 2 radiologists (F.E.D. and J.B.R., a neuroradiology fellow with an American Board of Radiology certification $)^{13,14}$ were noted: lesion location, maximal superior-inferior enhancement length (size [mm] and extent [number of vertebral body segments]), extent of T2 signal abnormality (number of vertebral segments), ratio of extent of T2 signal abnormality to contrast enhancement, and presence/absence of "rim" and "flame" signs, ${ }^{13} 2$ postgadolinium MR imaging findings specific for ISCM.

\section{Clinical Review}

The original clinical PET interpretations were reviewed to assess whether any of the following were reported: the $\operatorname{ISCM}(\mathrm{s})$, the primary neoplasm, additional metastatic disease (including specifically CNS, non-CNS, and skeletal spinal column metastases). Note that these clinical interpretations were issued by subspecialty trained nuclear medicine physicians at a large academic medical center. The time interval (days) between the PET and MR imaging examinations was calculated. For cases in which the PET report did not describe the ISCM(s) but PET occurred before the MR imaging, a review of the electronic medical record was performed to assess whether there was an impact on clinical care of the patient.

\section{Statistical Analysis}

PET and MR imaging findings were compared for each patient, in consensus by the 3 reviewers. Spinal segment localization on PET was considered concordant with that on MR imaging if at least a portion of the PET location overlapped with the MR imaging location. One-way $t$ test assuming unequal variances was performed to assess correlation of the following features with visibility of ISCMs on PET: number of days between MR imaging and PET and all aforementioned MR imaging features except for location. Contingency testing was performed to assess the relationship of the primary tumor pathology (lung cancer and melanoma) with the ability to detect ISCM on PET/CT. Bivariate linear coefficient analysis was performed to assess the correlation of the length of the ISCM on MR imaging (contrast enhancement) and on PET, by use of both the size measurement ( $\mathrm{mm}$ ) on MR imaging and longitudinal extent (number of vertebral segments) on both MR imaging and PET. Descriptive statistics were obtained by use of Excel 2010 (Microsoft Corporation, Redmond, Washington). Additional analyses were conducted with the use of SAS 

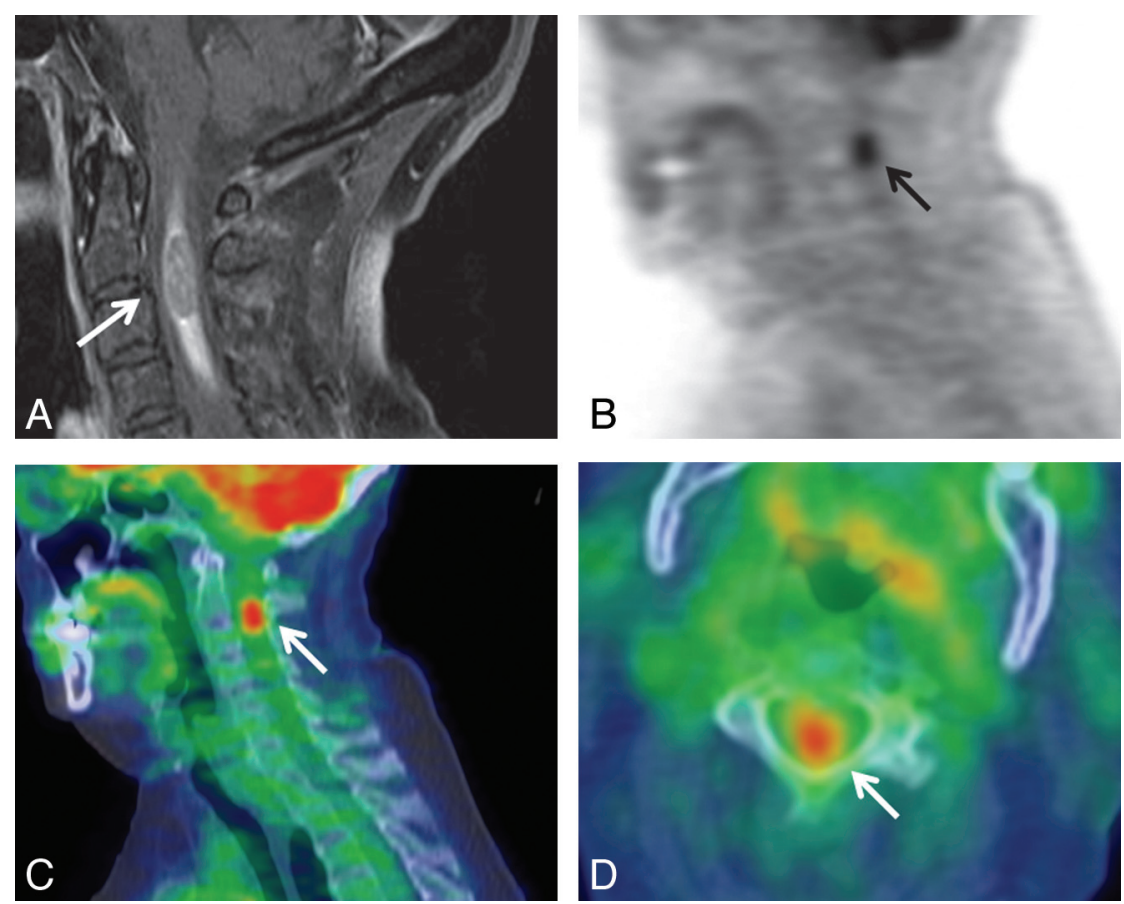

FIG 1. Solitary ISCM visible on PET. A 69-year-old woman (patient 8 in On-line Table 1) with non-small cell lung cancer underwent PET 13 days after MR imaging. A, Postcontrast fat-suppressed sagittal TIWI; $B$, sagittal PET only image; $C$, sagittal, and $D$, axial PET/CT fused images. The cervical cord ISCM was identified on original clinical and retrospective review. The location of the FDG avid ISCM in the upper cervical cord (C2-3) corresponds to the lesion location on MR imaging (C1-5) (arrows). SUVmax of the ISCM was 5.1 ( $2.0 \times$ that of blood pool). The clinical PET report favored a primary spinal cord neoplasm.

version 9.3 (SAS Institute, Cary, North Carolina). Statistical significance was defined as a value of $P<.05$.

\section{RESULTS}

\section{Summary of Subjects}

Of the 49 patients with 70 ISCMs and available pretreatment MR imaging, 17 (35\%) had a total of 32 PET examinations performed. Twenty-two PET examinations from 7 (41\%) of these 17 patients were excluded; 16 PET examinations were excluded because the PET examination was performed more than 60 days before the MR imaging and 6 because the PET was more than 14 days after the pretreatment MR imaging. The final study sample was $10 \mathrm{PET}$ examinations (9 PET/CT and 1 PET only) in 10 patients with 13 ISCMs. Patient individual and group characteristics are shown in On-line Tables 1 and 2, respectively. The mean age was 61 years (range, 37-75), and 6 (60\%) were female. The primary malignancies were lung cancer in 5 (50\%) patients (8 ISCMs) and melanoma in $5(50 \%)$ patients ( 5 ISCMs). Four of the 5 lung cancer patients had non-small cell carcinoma and 1 had small-cell lung carcinoma. Nine (90\%) patients had a solitary ISCM (Figs 1, 3, and 4$)$, whereas $1(10 \%)$ patient with lung cancer harbored multiple ISCMs (Fig 2).

The mean interval between MR imaging and PET was 11 days (range, 49 days before to 13 days after). PET occurred before or on the day of MR imaging in $8(80 \%)$ of patients. Other metastases were present on the PET report in 9 (90\%) patients; 7 (70\%) patients had non-CNS, nonspine metastases, 2 (20\%) patients had other CNS metastases, and 1 (10\%) patient had skeletal spinal column metastases reported (On-line Table 2).

\section{ISCM Findings on PET}

ISCM characteristics, including findings on PET, are displayed in On-line Tables 1 and 2, on an individual and group basis. On retrospective, blinded review of $\mathrm{PET}$, there was convincing evidence of 10 of $13(77 \%)$ ISCMs in 7 of $10(70 \%)$ patients (Figs 1-4). The location of FDG uptake for each of these 10 ISCMs was concordant with the location of the contrast-enhancing lesion on MR imaging (for example, Figs 1-4). For the 1 patient with multiple ISCMs, each ISCM was found and even 2 adjacent lesions at the T4 and T4-5 levels were discretely visualized on PET, as on MR imaging (Fig 2). Furthermore, the ISCM extent on PET (number of vertebral segments) correlated with the measured enhancing lesion size $(\mathrm{mm})$ on MR imaging $\left(R^{2}=0.803 ; P=.0004\right)$. The ISCM extent on PET did not correlate with the longitudinal extent of enhancement on MR imaging $(P=.151)$. The average SUVmax was 6.7 (range, 3.3-9.9). For each ISCM, the SUVmax of the lesion was greater than that of the mediastinal blood pool. The FDG avid lesions had round morphology in 8 (80\%) cases (for example, Figs 1, 2, and 4).

Three $(23 \%)$ ISCMs in 3 (30\%) patients could not be seen retrospectively on $\mathrm{PET}$, despite unblinding of reviewers to the MR imaging results. These 3 ISCMs were also not described in the original clinical PET interpretations. The PET examinations were performed 7 days before MR imaging in 2 of these patients and 21 days before MR imaging in another. In all 3 (100\%) patients, other metastases were reported on the original clinical PET interpretation (On-line Table 2). In none of the 10 patients did unblinding of reviewers to the MR imaging findings and PET report change the retrospective interpretation of the PET.

\section{Correlation of Clinical and MR Imaging Features with ISCM Visibility on PET}

There was no association between either the type of primary malignancy or the time interval between the MR imaging and PET and the visibility of ISCM on PET ( $P=.25, P=.7$, respectively). Three MR imaging features correlated with ISCM visibility on PET (On-line Table 2): 1) larger lesion enhancement size: mean size of lesions visible versus not visible on PET, $32.1 \mathrm{~mm}$ versus 6.0 $\mathrm{mm}$, respectively $(P=.038) ; 2$ ) larger extent of T2 signal abnormality: mean of 5.6 versus 1.0 segments $(P=.0081)$; and 3$)$ larger ratio of extent of T2 signal abnormality to contrast enhancement: 3.8 versus $1.0(P=.0069)$. MR imaging features that did not correlate with ISCM visibility on PET were extent of lesion contrast enhancement (number of vertebral segments) $(P=.11)$ and the presence of the "rim" sign $(P=.50)$, "flame" sign $(P=.50)$, either $\operatorname{sign}(P=.23)$, or both signs $(P=.53)$. 

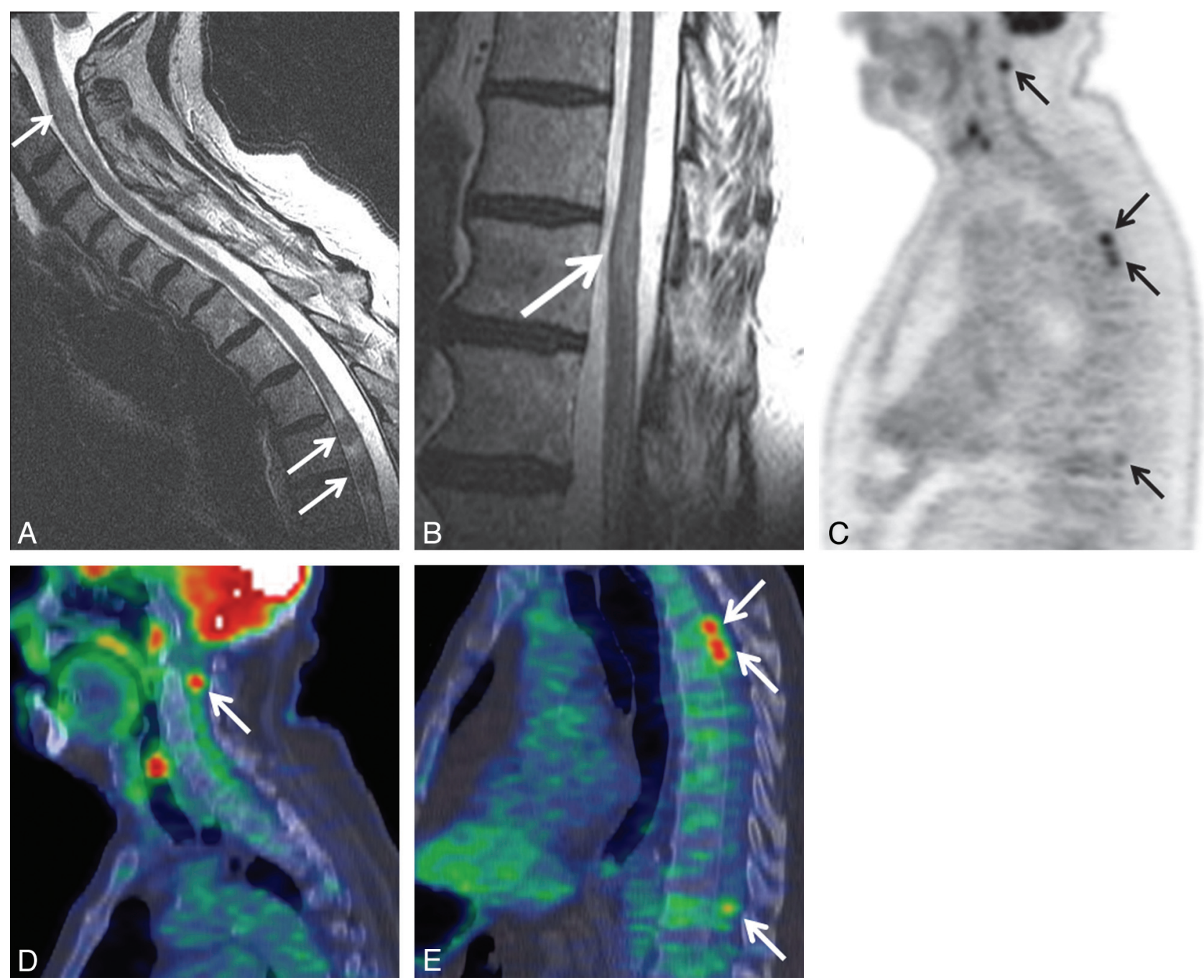

FIG 2. Patient with multiple ISCMs, all visible on PET. A 73-year-old man (patient 5 in On-line Table 1) with non-small cell lung cancer underwent PET 5 days before MR imaging. Precontrast sagittal T2WI of the cervicothoracic (A) and lower thoracic (B) regions; $C$, sagittal entire spine PET only image; sagittal cervicothoracic (D) and thoracic (E) region PET/CT fused images. Several cervical and thoracic cord ISCMs were identified on original clinical and retrospective review of the PET examination, including 2 immediately adjacent metastases at T4 and T4-5. The locations on MR imaging (C2; T4; T4-5; T11) correlate with those on PET (Cl-2; T4; T5; T10) (arrows). SUVmax of the ISCMs ranged from 5.3-9.9 (2.9-5.5 $\times$ that of blood pool). (Note: Postcontrast sagittal TIWI from the same patient showing enhancement of the ISCMs is included in Reference 14, Fig 1, in which the figure emphasized the asymptomatic status of some patients with ISCM).

\section{Clinical Reporting of ISCMs on PET}

Of the 10 patients with 13 ISCMs, ISCMs were confidently prospectively reported in the original clinical PET report in $2(20 \%)$, accounting for 5 of 13 (38\%) ISCMs (for example, Fig 2). In 1 patient, a cord lesion was reported, but a primary cord neoplasm was favored, at least in part because of lack of other evidence of FDG metastatic disease (Fig 1). In 7 of 10 (70\%) patients with 7 of 13 (54\%) ISCMs, the ISCMs were not reported (Figs 3 and 4). In 4 of these 7 (57\%) patients and 4 of 7 (57\%) ISCMs, the ISCMs were identified on blinded, retrospective review (Figs 3 and 4). These ISCMs ranged in size from 7-114 mm on MR imaging. The PET examinations were performed 49 days and 1 day prior in 2 patients, on the day of MR imaging in 1 patient, and 7 days after MR imaging in 1 patient. Of these 4 ISCMs visible on retrospective review but not clinically reported, the lack of identification on PET probably had clinical impact in 1 patient. The patient shown in Fig 3 had PET performed while having lower extremity symptoms. It was not until the MR imaging 49 days later that the ISCM of the conus with leptomeningeal spread in the cauda equina was formally diagnosed and subsequently treated with steroids and radiation.

\section{DISCUSSION}

The present study shows that most ISCMs can be detected on PET when this is performed near the time of diagnostic MR imaging. $\mathrm{PET}$ is often performed prior to spine MR imaging and may thus be the first imaging study to reveal an ISCM. However, ISCMs may not be clinically reported on PET, as the minority of ISCMs in the minority of patients had been confidently clinically reported in this study. This can have clinical impact. Larger lesions with more edema are more likely to be visible on PET, but even subcentimeter ISCMs can be detected.

These results are clinically relevant in several ways. We hope that these results will stimulate those who read PET primarily to focus on the spinal cord as part of their search pat- 

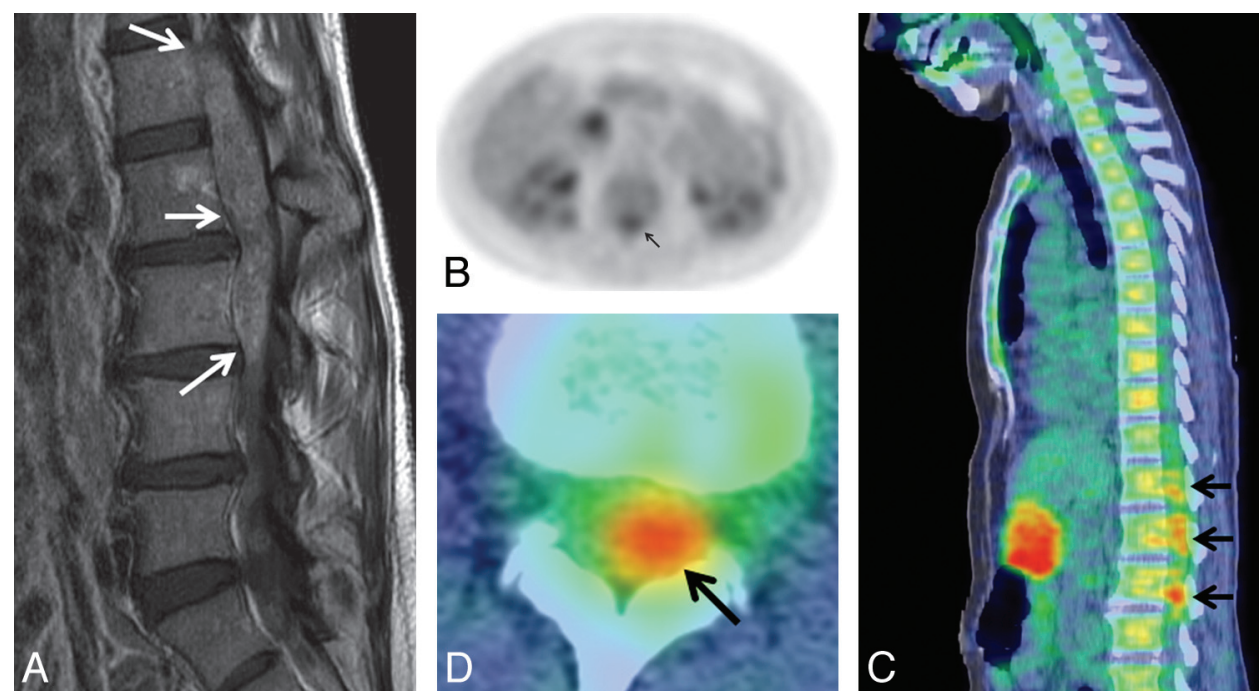

FIG 3. ISCM not originally clinically reported, probably with clinical impact. A 57-year-old woman (patient 2 from On-line Table 1) with melanoma underwent PET 49 days before MR imaging. $A$, Postcontrast sagittal TIWI; $B$, axial PET only image; $C$, sagittal and $D$, axial PET/CT fused images. The fusiform, patchy lower spinal canal uptake (conus and cauda equina region) was not described in the original clinical PET report. It was not until the MR imaging nearly 2 months later that the large exophytic ISCM arising from the conus was formally diagnosed and treated. Note that only a single sagittal PET/CT section is shown; on review of multiple adjacent images; the PET uptake appears contiguous. This is the largest ISCM in this series. The location of enhancing lesions on MR imaging (L1-4) corresponds with PET uptake (T12/L1-L3/4) (arrows). SUVmax was $5.4(3.0 \times$ that of blood pool).
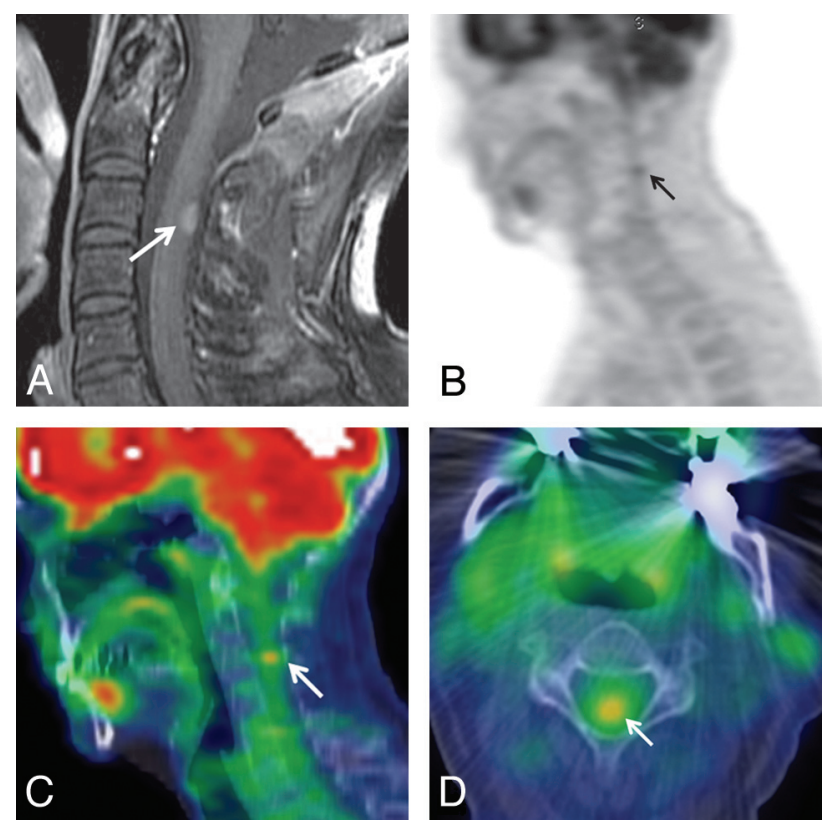

FIG 4. Smallest ISCM visible on PET, not clinically reported. A 54year-old woman (patient 9 from On-line Table 1) with non-small cell lung cancer underwent PET 1 day before MR imaging. $A$, Postcontrast sagittal TIWI; $B$, sagittal PET only image; $C$, sagittal, and $D$, axial PET/CT fused images. A small, 7-mm cervical ISCM (arrows) was not described on the original clinical PET report. The location of the FDG avid ISCM in the upper cervical cord (C3-4) corresponds to the lesion location on MR imaging (C3) (arrows). The SUVmax was $3.3(1.9 \times$ that of blood pool).

tern. Missing ISCMs can delay diagnosis, and identifying and treating ISCM(s) early improves outcomes. ${ }^{15,16}$ The fact that ISCMs can be asymptomatic or present with minimal neuro-

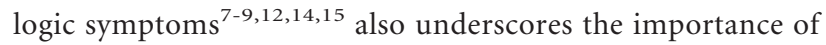
detecting the lesions on early imaging, even when other evidence of metastatic disease is present, as there was for most patients in the current study. Similarly, the results of our study should encourage those who do not interpret PET primarily but correlate with it when interpreting MR imaging to actually review the PET images, not just the interpretation. Finally, PET may be considered as an alternative to MR imaging for the evaluation for ISCMs in certain settings, including in patients for whom MR imaging is contraindicated and those already undergoing PET for disease staging. Patients suspected of having ISCM with equivocal spinal cord findings on MR imaging who cannot receive intravenous gadolinium may also be good candidates for PET.

Our study adds to the existing literature describing PET findings of ISCMs, which is otherwise limited to case reports. ${ }^{7-12}$ This is the first study starting with a large number of ISCMs identified on MR imaging to evaluate the conspicuity of ISCMs on PET and how visibility on PET correlates with MR imaging features. Note that in a recent neuroimaging study assessing PET findings of 9 intramedullary spinal cord tumors, only primary spinal cord tumors were included; primary tumors can also demonstrate increased uptake on PET. ${ }^{17}$

There are several possible reasons for nondiagnosis and the low clinical identification rate of ISCMs on the original PET reports for patients in the current study. These probably include lack of specific attention to the spinal cord, lack of familiarity with the appearance or even their existence (as these lesions are rare), confounding or distracting uptake in the immediately adjacent vertebral column, satisfaction of search (as other sites of FDG avid metastatic disease were often present), and the small size of some of the lesions. As part of the CNS, the spinal cord may also be overlooked on PET just as the intracranial contents are often not well scrutinized on this technique. Specifically, MR imaging has been shown to have superior sensitivity and specificity in detecting intracranial metastases, even in FDG avid malignancies. ${ }^{18,19}$ Contributory factors are that inherent phys- 
iologic FDG uptake within the brain limits visualization of superimposed FDG avid lesions and that MR imaging has superior spatial sensitivity. Moreover, Lee et $\mathrm{al}^{20}$ showed that approximately one-third of lung cancer brain metastases appeared hypometabolic on PET.

Limitations of this study include those inherent to a retrospective study. The time span over which data were collected leads to differences in the MR imaging and PET imaging techniques. Another limitation is that the MR imaging and PET images were interpreted by consensus review and despite blinding, readers were aware that there was at least 1 ISCM in each case. Although the exclusion criteria limiting the time interval between PET and MR imaging examinations may have eliminated some patients who harbored an ISCM that could have been visible on PET, we chose those criteria to minimize the possibility of "false-negative" PET examinations as the result of an ISCM either not yet having developed or already having been treated. Additionally, 10 patients with 13 ISCMs is a relatively small series, but this is the largest such series to be published to our knowledge. Some factors may overestimate the sensitivity of PET for ISCMs, such as the fact that the consensus reviewers knew at least 1 ISCM was present in each case; others may underestimate, such as the fact that not all malignancies are FDG avid.

Future work may include studying a larger patient population, potentially across multiple centers, to allow further identification of factors that affect ISCM conspicuity on PET. Some small ISCMs in this series could be detected on PET, whereas others could not, and we could not discover an explanation for this in this current work. Identifying such tumor or patient characteristics may help determine patients for whom PET is or is not a reliable screening tool for ISCMs.

\section{CONCLUSIONS}

We describe the PET findings from a large series of ISCMs and correlate these with MR imaging features. The results should be helpful to radiologists. Most ISCMs are visible on PET when this is performed near the time of diagnostic MR imaging, but they may not be clinically reported on PET. Larger lesions with more edema are more likely to be visible on PET. The spinal cord should be specifically and carefully assessed on PET for evidence of ISCMs to provide timely and accurate diagnosis. In some settings, such as when MR imaging is contraindicated, PET may be considered as an option to evaluate for ISCM.

\section{REFERENCES}

1. Chason JL, Walker FB, Landers JW. Metastatic carcinoma in the central nervous system and dorsal root ganglia: a prospective autopsy study. Cancer 1963;16:781-87
2. Costigan DA, Winkelman MD. Intramedullary spinal cord metastasis: a clinicopathological study of 13 cases. J Neurosurg 1985;62:227-33

3. Edelson RN, Deck MD, Posner JB. Intramedullary spinal cord metastases: clinical and radiographic findings in nine cases. $\mathrm{Neu}$ rology 1972;22:1222-31

4. Lee SS, Kim MK, Sym SJ, et al. Intramedullary spinal cord metastases: a single-institution experience. J Neurooncol 2007;84:85-89

5. Reddy SC, Vijayamohan G, Rao GR. Delayed CT myelography in spinal intramedullary metastasis. $J$ Comput Assist Tomogr 1984;8:1182-85

6. Sze G, Krol G, Zimmerman RD, et al. Intramedullary disease of the spine: diagnosis using gadolinium-DTPA-enhanced MR imaging. AJR Am J Roentgenol 1988;151:1193-204

7. Jayasundera MV, Thompson JF, Fulham MJ. Intramedullary spinal cord metastasis from carcinoma of the lung: detection by positron emission tomography. Eur J Cancer 1997;33:508-09

8. Komori T, Delbeke D. Leptomeningeal carcinomatosis and intramedullary spinal cord metastases from lung cancer: detection with FDG positron emission tomography. Clin Nucl Med 2001;26:905-07

9. Nguyen NC, Sayed MM, Taalab K, et al. Spinal cord metastases from lung cancer: detection with F-18 FDG PET/CT. Clin Nucl Med 2008;33:356-58

10. Jeon MJ, Kim TY, Han JM, et al. Intramedullary spinal cord metastasis from papillary thyroid carcinoma. Thyroid 2011;21:1269-71

11. Pellegrini D, Quezel MA, Bruetman JE. Intramedullary spinal cord metastasis. Arch Neurol 2009;66:1422

12. Poggi MM, Patronas N, Buttman JA, et al. Intramedullary spinal cord metastasis from renal cell carcinoma: detection by positron emission tomography. Clin Nucl Med 2001;26:837-39

13. Rykken JB, Diehn FE, Hunt $\mathrm{CH}$, et al. Rim and flame signs: postgadolinium MRI findings specific for non-CNS intramedullary spinal cord metastases. AJNR Am J Neuroradiol 2013;34:908-15

14. Rykken JB, Diehn FE, Hunt CH, et al. Intramedullary spinal cord metastases: MRI and relevant clinical features from a 13-year institutional case series. AJNR Am J Neuroradiol 2013;34:2043-49

15. Sung WS, Sung MJ, Chan JH, et al. Intramedullary spinal cord metastases: a 20-year institutional experience with a comprehensive literature review. World Neurosurg 2013;79:576-84

16. Kalayci M, Cagavi F, Gul S, et al. Intramedullary spinal cord metastases: diagnosis and treatment: an illustrated review. Acta Neurochir 2004;146:1347-54

17. Tomura $\mathrm{N}$, Ito $\mathrm{Y}$, Matsuoka $\mathrm{H}$, et al. PET findings of intramedullary tumors of the spinal cord using [18F] FDG and [11C] methionine. AJNR Am J Neuroradiol 2013;34:1278-83

18. Krüger S, Mottaghy FM, Buck AK, et al. Brain metastasis in lung cancer: comparison of cerebral MRI and 18F-FDG-PET/CT for diagnosis in the initial staging. Nuklearmedizin 2011;50:101-06

19. Pfannenberg C, Aschoff P, Schanz S, et al. Prospective comparison of 18F-fluorodeoxyglucose positron emission tomography/ computed tomography and whole-body magnetic resonance imaging in staging of advanced malignant melanoma. Eur J Cancer 2007;43:557-64

20. Lee HY, Chung JK, Jeong JM, et al. Comparison of FDG-PET findings of brain metastasis from non-small-cell lung cancer and smallcell lung cancer. Ann Nucl Med 2008;22:281-86 